\title{
Avaliação do perfil demográfico e do estado funcional pós cirurgia de Latarjet em pacientes com luxação redicivante do ombro
}

\author{
Evaluation of the demographic profile and the functional state after Latarjet surgery in \\ patients with recurrent shoulder dislocation
}

Evaluacíon del perfil demográfico y del estado funcional después de la cirugía Latarjet em pacientes com dislocación de hombro recurrente

Jones Aguiar de Azevedo ${ }^{\text {* }}$, Rafael Patrocínio de Paula Costa1, Marcos André Araújo Accioly Filho, José Atualpa Pinheiro Júnior ${ }^{1}$, Fabiana Maria Oliveira Freitas ${ }^{1}$, Ravenna Cavalcante Bessa de Queiroz ${ }^{1}$, Raimundo Nogueira Oliveira Neto'1.

\section{RESUMO}

Objetivo: Avaliar a funcionalidade de pacientes portadores de luxação recidivante do ombro submetidos à cirurgia de Latarjet em um hospital escola no Ceará, entre março de 2017 e agosto de 2019, caracterizando o perfil sociodemográfico dos pacientes avaliados. Métodos: Pesquisa documental a partir de prontuários e análise clínica retrospectiva dos pacientes selecionados. Realizada avaliação comparativa do estado funcional pré-tratamento e, posteriormente, pós-tratamento instituído, por meio de aplicação do questionário e avaliação de escore de Carter-Rowe. Resultados: O estudo epidemiológico foi composto, em geral, de um grupo de pacientes predominantemente do sexo masculino, destes $40 \%$ com idade igual ou inferior a 40 anos, praticantes de atividades físicas ou trabalhadores braçais. A avaliação comparativa de escore em estudo demonstrou que há melhora no resultado funcional destes pacientes. Cerca de $90 \%$ dos pacientes em estudo obtiveram escore de Carter-Rowe com pontuações acima de 75 (setenta e cinco) no período pósoperatório, denotando o bom resultado com a terapêutica utilizada. Conclusão: O procedimento cirúrgico descrito por Latarjet notadamente apresenta-se como uma alternativa terapêutica adequada à população estudada, resultando em melhora funcional dos pacientes estudados e diminuição do quadro de instabilidade.

Palavras-chave: Articulação do ombro, Luxação do ombro, Lesões de Bankart.

\begin{abstract}
Objective: Evaluate the functionality of patients with recurrent shoulder dislocation who underwent Latarjet surgery in a teaching hospital in Ceará, between March 2017 and August 2019, characterizing the sociodemographic profile of the evaluated patients. Methods: Documentary research from medical records and retrospective clinical analysis of selected patients. Comparative assessment of the functional status before and after treatment was carried out, through the application of the questionnaire and assessment of the CarterRowe score. Results: The epidemiological study was composed, in general, of a group of predominantly male patients, of which $40 \%$ were aged 40 years or less, practitioners of physical activities or manual workers. The comparative evaluation of the score under study showed that there is an improvement in the functional outcome of these patients. About $90 \%$ of the patients in the study had a Carter-Rowe score with scores above 75 (seventy-five) in the postoperative period, denoting the good result with the therapy used. Conclusion: The surgical procedure described by Latarjet notably presents itself as an adequate therapeutic alternative for the studied population, resulting in functional improvement of the studied patients and reduction of instability.
\end{abstract}

Key words: Shoulder joint, Shoulder dislocation, Bankart lesions.

${ }^{1}$ Universidade Federal do Ceará (UFC), Fortaleza - CE. *E-mail: jones.aa.42@gmail.com 


\section{RESUMEN}

Objetivo: Evaluar la funcionalidad de pacientes con luxación recurrente de hombro intervenidos de Latarjet en un hospital universitario de Ceará, entre marzo de 2017 y agosto de 2019, caracterizando el perfil sociodemográfico de los pacientes evaluados. Métodos: Investigación documental a partir de historias clínicas y análisis clínico retrospectivo de pacientes seleccionados. Se realizó una evaluación comparativa del estado funcional antes y después del tratamiento, mediante la aplicación del cuestionario y la evaluación del puntaje de Carter-Rowe. Resultados: El estudio epidemiológico estuvo compuesto, en general, por un grupo de pacientes predominantemente del sexo masculino, de los cuales el $40 \%$ eran de 40 años o menos, practicantes de actividades físicas o trabajadores manuales. La evaluación comparativa de la puntuación en estudio mostró que existe una mejora en el resultado funcional de estos pacientes. Aproximadamente el $90 \%$ de los pacientes del estudio tenían una puntuación de Carter-Rowe con puntuaciones superiores a 75 (setenta y cinco) en el postoperatorio, lo que denota el buen resultado con la terapia utilizada. Conclusión: El procedimiento quirúrgico descrito por Latarjet se presenta notablemente como una alternativa terapéutica adecuada para la población estudiada, resultando en una mejora funcional de los pacientes estudiados y una reducción de la inestabilidad.

Palabras clave: Articulación del hombro, Luxación del hombro, Lesiones de Bankart.

\section{INTRODUÇÃO}

O ombro é uma articulação complexa e com amplitude de movimento ampla. É composta pela face articular da glenóide, em formato côncavo, e pela face articular da porção proximal do úmero, em formato esférico e convexo. Entre as faces articulares existe um espaço virtual, ou seja, constituído por pequena quantidade de líquido sinovial (BAKHSH W e NICANDRI G, 2018; MOURA DL, et al., 2018).

O contato articular da cabeça umeral sobre a glenóide descreve um trajeto a ser percorrido, conhecido pelo termo "track" glenoidal. A lesão de Hill-Sachs, descrita como fratura por impacção em região posterior de cabeça umeral, pode estar, durante a movimentação do ombro, em contato com este trajeto, lesões "on track" ou fora dele, lesões "off track". As lesões sem contato com o "track" glenoidal têm maior risco de luxação e "engaging" entre a falha óssea umeral e a glenoide (ITOI E, 2017).

A articulação do ombro detém um movimento tridimensional, permitindo a maior amplitude de movimento dentre as articulações do corpo humano. No entanto, apresenta um maior potencial de instabilidade que as demais. A estabilidade da articulação do ombro depende de algumas estruturas e de certas condições, como a congruência das superfícies, a integridade dos ligamentos glenoumerais, da cápsula, do labrum e do sistema muscular, como a musculatura do manguito rotador, deltóide e peitoral maior (BAKHSH W e NICANDRI G, 2018).

O manguito rotador é uma estrutura tendínea composta pelos músculos supraespinhal, infraespinhal, redondo menor e subescapular. Estes tendões unidos formam uma cobertura sobre a cabeça umeral com função de auxiliar na mobilidade, proteção mecânica e na estabilidade da articulação do ombro (MOURA DL, et al., 2018; LIZZIO VA, et al., 2017).

A instabilidade glenoumeral ocorre por falha em algum desses fatores e apresenta um amplo espectro clínico de acometimento. Uma complicação importante relacionada aos quadros de instabilidade glenoumeral é a luxação recidivante do ombro. Esta afecção ocorre em cerca de $60 \%$ dos pacientes, e tem como razão a falha na cicatrização ligamentar ou de alteração estrutural na cobertura óssea da glenoide ou capsular (NA VVG, et al., 2016).

A luxação recidivante do ombro é uma afecção caracterizada pela ocorrência de episódios recorrentes da perda de congruência desta articulação. Este quadro pode afetar as atividades de vida diária do paciente, limitando-as, principalmente no que se refere a realizar movimentos de abdução e rotação externa do membro afetado, pois estes predispõem à eventual luxação. O risco de haver recorrências em luxações pode ser estimado pela avaliação do perfil de paciente e, de forma sucinta, por ferramentas, como "Predicting Recurrent Instability of the Shoulder" (PRIS) (OLDS M, et al., 2020). 
Essa condição, quando de etiologia traumática, é associada à lesão da cápsula articular, de ligamentos, do labrum ou de superfície óssea e aumenta a chance de o portador destas lesões necessitar de terapêutica cirúrgica. Em pacientes com mais de 40 anos, além das lesões previamente citadas, há uma maior prevalência de ruptura de tendões do manguito rotador (MOURA DL, et al., 2018; LIZZIO VA, et al., 2017; SILVA LA, et al., 2015).

Em caso de lesão ósseas, algumas técnicas cirúrgicas foram descritas, uma das intervenções mais utilizadas para o tratamento é a técnica de Latarjet, via acesso deltopeitoral, entre o músculo deltóide e músculo peitoral maior e consiste na transferência de enxerto do processo coracoide para face anterior da glenoide e fixação com parafusos 3,5 mm (GETZ CL e JOYCE CD, 2020).

Essa técnica tem como princípio, o triplo bloqueio. O bloqueio ósseo, determinado pelo enxerto do coracoide, aumentando a superfície da glenoide, há o efeito "sling" realizado pelo tendão conjunto, composto pela cabeça curta do bíceps braquial e coracobraquial, inserido na ponta do coracoide e o bloqueio ligamentar decorrente da sutura do ligamento coracoacromial junto a capsula articular (WOODMASS JM, et al., 2019).

Além da transferência do processo coracoide, para pacientes com perda óssea glenoidal, pode-se utilizar enxerto ósseo de crista ilíaca e, baseado nos escores funcionais, manter similaridade na performance clínica observada entre pacientes submetidos a essas duas diferentes técnicas (MORODER P, et al., 2019).

Durante o seguimento pós-operatório, é orientado ao paciente o uso de uma tipoia de velpeau durante os primeiros 15 dias, liberando os movimentos do cotovelo, punho e mão. No decorrer das duas primeiras semanas, estimula-se a realização de movimentos de elevação e rotação externa, sendo liberadas atividades de vida diária com parcimônia a partir do decimo quinto dia e, com 01 mês de pós-operatório, se inicia 0 programa de fortalecimento muscular dos membros superiores.

O interesse em avaliar a funcionalidade e o estado pós terapêutica motivou diversos pesquisadores a confeccionarem escores de pontuação e questionários. O Questionário Rowe, primeiramente descrito em 1978, tem objetivo de avaliar o resultado pós cirúrgico do tratamento para instabilidade anterior do ombro. O valor máximo atribuído são 100 pontos, sendo o intervalo 91 a 100 como resultado excelente e ruim o intervalo com valor menor ou igual a 50. Resultados intermediários entre 89 e 75 pontos recebe a classificação boa e resultados entre 74 e 51 pontos recebe a classificação regular (MARCONDES FB, et al., 2011).

Desta forma, dada a complexidade das patologias do ombro e da variedade terapêutica descrita em literatura médica, no intuito de padronização de condutas ortopédicas em hospital universitário e do melhor entendimento do perfil de pacientes, justifica-se a realização deste estudo que foi desenhado com o objetivo de avaliação dos pacientes e do resultado funcional durante seguimento ambulatorial continuado destes, e da descrição e perfil demográfico da população em estudo.

\section{MÉTODOS}

O estudo incluiu pacientes, acompanhados no ambulatório de Ortopedia em hospital escola no Ceará, com o diagnóstico de luxação recidivante do ombro de etiologia traumática e que foram submetidos a tratamento cirúrgico pela técnica descrita por Latarjet, no período compreendido entre março de 2017 e agosto de 2019. Foram excluídos do trabalho os pacientes que ainda não realizaram o tratamento e aqueles que o fizeram por meio de outra técnica cirúrgica.

Para a avaliação do estado funcional dos pacientes antes e após o tratamento cirúrgico, foi optado pela utilização do questionário Carter-Rowe, consagrado em literatura médica internacional para avaliação ambulatorial de funcionalidade e instabilidade do ombro. Este questionário foi traduzido, adaptado para a língua portuguesa e validado para o uso na última década (MARCONDES FB, et al., 2011).

A coleta de dados foi realizada após aprovação do estudo pelo Cômite de Ética e Pesquisa (CEP), com liberação do certificado de apresentação de apreciação ética (CAAE) com o número 25379719.1.0000.5045. Realizou-se pesquisa documental a partir dos prontuários médicos, com 0 intuito de produzir a análise retrospectiva dos prontuários dos pacientes selecionados, observando o estado funcional que precedia 0 tratamento, e atribuindo uma pontuação a este estado por meio do escore de Carter-Rowe. 
Concluída esta etapa, a amostra selecionada foi contactada e convidada para realização de entrevista, que incluiu a coleta de dados sociodemográficos (idade, ocupação, sexo e prática de atividades esportivas) e a aplicação do questionário de Carter-Rowe. Os dados sociodemográficos coletados foram organizados para a compreensão a respeito da população afetada pela luxação recidivante do ombro em nosso local de estudo. O uso da ferramenta supracitada de análise quantitativa possibilitou melhor avaliação e entendimento da estabilidade, da mobilidade e da função do ombro propriamente dita.

O projeto foi submetido à Plataforma Brasil vinculado ao comitê de ética do hospital universitário, respeitando-se as normas da resolução Número 466/2012 do Conselho Nacional de Saúde, a qual rege o processo de desenvolvimento de pesquisas com seres humanos (BRASIL, 2012). Após a aprovação, realizouse a pesquisa documental e a convocação dos participantes, com apresentação do Termo de Consentimento Livre e Esclarecido. Foram feitas referências a liberdade de recusa, assim como a interrupção de sua participação em qualquer etapa do estudo. Foi garantido o sigilo e o anonimato na apresentação dos resultados.

\section{RESULTADOS}

Foram avaliados 29 pacientes, dos quais permaneceram apenas 10 na amostra final. 2 indivíduos foram excluídos do estudo por terapêutica diferente à proposta em metodologia. 17 pacientes não compareceram as reuniões clínicas e entrevistas. A análise de dados permitiu observar que a população acometida era composta por $80 \%$ de pacientes do gênero masculino e apenas $20 \%$ do feminino, sendo pacientes adultos jovens, $60 \%$ com menos de 40 anos de idade. Como observado nos dados alocados na Tabela 1, apenas $40 \%$ praticavam algum tipo de atividade física.

Tabela 1 - Dados epidemiológicos da amostra selecionada.

\begin{tabular}{ccc}
\hline Variável & N & $\%$ \\
\hline Sexo & & \\
\hline Masculino & 8 & 80 \\
\hline Feminino & 2 & 20 \\
\hline Idade & & \\
\hline$<$ ou $=40$ & 6 & $60 \%$ \\
\hline$>40$ & 4 & $40 \%$ \\
\hline Atividade física & & \\
\hline Praticantes & 4 & $40 \%$ \\
\hline Não praticantes & 6 & $60 \%$ \\
\hline Total & $\mathbf{1 0 0}$ & - \\
\hline
\end{tabular}

Fonte: Azevedo JA, et al., 2021.

O escore de Carter-Rowe avalia três aspectos: a função, a mobilidade e a estabilidade, graduando a pontuação em cada um destes tópicos. Os escores de Carter-Rowe, no estado prévio ao tratamento, descrevem pontuações compreendidas entre os estágios funcionais ruim e regular. A avaliação do escore no estado pós terapêutico nota-se uma melhora do nível funcional de toda a população em estudo. No resultado final, $30 \%$ apresentaram resultado excelente com pontuação acima de 90 pontos e $60 \%$ com pontuação entre 75 a 90 pontos (Tabela 2).

Os pacientes apresentaram melhora da funcionalidade do ombro afetado e retornaram as atividades de vida diária e ocupacionais, mas alguns deles apresentavam discreta limitação de rotação externa. Alguns pacientes apresentaram ainda uma apreensão residual a amplitude de movimento extrema, como abdução e rotação externa, mas como relatado pelos mesmos, isto não limitava as suas atividades de vida diária. 
Tabela 2 - Escore funcional Carter-Rowe avaliada na situação pré-operatória e pós-operatória.

\begin{tabular}{lcc}
\hline Variável & $\mathbf{N}$ & $\%$ \\
\hline Escore Carter-Rowe (pré-operatório) & & \\
\hline $74-51$ (Regular) & 6 & 60 \\
\hline$<$ ou igual a 50 (Ruim) & 4 & 40 \\
\hline Escore Carter-Rowe (pós-operatório) & & \\
\hline$>90$ & 3 & 30 \\
\hline $75-90$ & 6 & 60 \\
\hline $51-74$ & 1 & 10 \\
\hline$<51$ & 0 & 0 \\
\hline Total & $\mathbf{1 0}$ & - \\
\hline
\end{tabular}

Fonte: Azevedo JA, et al., 2021.

A análise pré-operatória permitiu observar que os pacientes apresentavam perda de pontos, em sua maior percentagem, nos aspectos estabilidade e função. Apenas $20 \%$ apresentavam dor moderada na avaliação pré-cirúrgica.

\section{DISCUSSÃO}

A avaliação especializada do paciente que desenvolve a instabilidade do ombro com episódios recorrentes de luxação é mandatória para um bom planejamento terapêutico. A avaliação inicia-se com a investigação acerca do primeiro episódio e a devida caracterização do evento, associada a informações como a idade, o membro afetado, a prática regular de atividades esportivas, a presença de comorbidade e os movimentos com maior apreensão, caracterizada por sensação de iminência de luxação articular e dor. A anamnese precisa e com o esclarecimento destes questionamentos auxilia no diagnóstico, no planejamento terapêutico e na avaliação de resultados (LIZZIO VA, et al., 2017).

Após a realização de história clínica, o exame físico nos traz informações relevantes. Primeiro é realizada a inspeção da região afetada em comparativo ao lado contralateral a fim de observar assimetrias musculares, zonas de edema e a altura dos ombros. Após, ao avaliar a amplitude de movimento, é importante testar a elevação, a extensão, a abdução, a adução e as rotações e, por meio disso, é possível observar se o examinado apresenta alguma limitação, principalmente, dos movimentos de rotações (BAKHSH W e NICANDRI G, 2018).

O exame deve prosseguir com a realização dos testes específicos para esta afecção ou manobras provocativas. Há a necessidade de avaliação da integridade dos tendões do manguito rotador. $O$ teste do sulco e o teste de Gagey apresentam o objetivo de avaliar se o paciente detém um estado de frouxidão ligamentar, podendo esta ser a etiologia do quadro de instabilidade (ESHOJ H, et al., 2018).

O teste de apreensão nos fornece a informação sobre o grau de instabilidade que o enfermo apresenta, esta manobra consiste na realização de uma abdução associada a rotação externa e o paciente irá responder se há sensação de luxação da articulação, é realizado com zero graus, quarenta e cinco graus e noventa graus, caso seja evidenciando apreensão mesmo ao mínimo grau de abdução, isto denota que há a presença de uma lesão de maior gravidade (LIZZIO VA, et al., 2017).

Após a avaliação inicial por meio da anamnese e do exame físico, a avaliação complementar é iniciada com a realização de radiografias do ombro afetado nas incidências anteroposterior verdadeira, sob correção de 45 de rotação escapular, perfil escapular e a incidência Stryker, tecnicamente realizada com o paciente em decúbito dorsal sobre o chassi, realizando flexão de ombro a ser examinado, mão ipsilateral ao ombro examinado sobre a cabeça e ampola de raio $x$ direcionada $10^{\circ}$ com orientação cefálica (SANTOS PD, et al.,1988). 
Estes exames de imagem permitem ao ortopedista avaliar e confirmar a presença de lesões, como a associação de lesão óssea e do labrum glenoidal antero-inferior, conhecida como lesão de Bankart, e a lesão de Hill-Sachs.

A avaliação complementar possibilita a correlação clínico-radiológica e elaboração de diagnóstico presuntivo. A tomografia computadorizada é indicada quando há necessidade de uma melhor caracterização da lesão durante o planejamento cirúrgico e a ressonância magnética é indicada na avaliação de lesões labrais, ligamentares e lesões associadas, como ruptura tendões do manguito rotador ou cabo longo do bíceps.

O tratamento desta condição após todo o processo diagnóstico irá variar de acordo com a etiologia da afecção. Quando a instabilidade provém de uma etiologia atraumática, no caso dos pacientes que apresentam quadro associado de hiperfrouxidão ligamentar, a conduta inicial mais indicada seria a intervenção conservadora por meio de fisioterapia para o fortalecimento da musculatura periarticular, alongamento tendíneo, ganho de propriocepção e analgesia, caso algum quadro álgico associado. Em contrapartida, quando o quadro de luxação recidivante do ombro é de etiologia traumática, maior percentagem dos casos, a conduta exige intervenção cirúrgica.

A luxação glenoumeral anterior é influenciada por inúmeros fatores, destes, os mais importantes se referem à idade do paciente no momento do primeiro episódio, ao sexo, à participação em atividades esportivas que necessitam de contato físico, à associação do membro lesionado com a dominância, à presença de lesão óssea ou lesão labral isolada. Dentre os fatores avaliados, a decisão terapêutica é baseada, principalmente no sexo, dominância e grau de atividades do indivíduo. Além disso, a caracterização da lesão é mandatória, sendo perdas ósseas superiores a $20 \%$ compatíveis com propedêutica cirúrgica (LAU BC, et al., 2021).

A população acometida apresentava semelhanças à literatura quanto ao gênero acometido e à sua proporção, assim como a faixa etária acometida. No entanto, difere no aspecto da prática esportiva. O perfil de pacientes na literatura varia de acordo com o local do estudo, com prevalência do sexo masculino e de praticantes de atividade física em cerca de 75 \% da população estudada (SILVA LA, et al., 2015).

A partir da análise dos dados, foi possível verificar que os pacientes apresentaram um intervalo maior que cinco anos, desde o primeiro evento até o momento da terapêutica proposta, denotando, nesta informação, a dificuldade $o$ atraso no trajeto do paciente da atenção primária ao serviço terciário para avaliação por um especialista. Avaliamos a ocupação de cada paciente e podemos interpretar que os pacientes acometidos no trabalho realizavam serviços que exigiam uma alta carga e, em alguns deles, movimentos que exigiam elevação do membro superior acima de noventa graus.

A limitação de rotação externa é uma das principais complicações. É importante relatar que todos os casos analisados apresentavam instabilidade de etiologia traumática e que, durante o período de seguimento, também não foram observados casos de recidiva pós cirurgia e nem casos que necessitaram de intervenção para revisão (SILVA LA, et al., 2015).

Assim como o que foi constatado neste estudo, Carrazzone OL, et al. (2010) avaliou a prevalência de pacientes com quadro de luxação recidivante do ombro em uma população específica e notou a prevalência de paciente jovens com menos de 40 anos de idade, apresentando a média de 27 anos e, em sua maioria, não praticantes de atividade esportiva. Moura DL, et al. (2018), em seu estudo, observou que o tratamento cirúrgico de pacientes com esta afecção por meio do uso da técnica de Latarjet, apresentou um excelente resultado funcional com maior segurança para retorno às atividades laborais e atividades esportivas de contato sem relato de recidiva do quadro, demonstrando um desfecho satisfatório.

Atualmente, ganham notoriedade as cirurgias artroscópicas do ombro, incluindo os tratamentos de instabilidade glenoumeral. A técnica de Larjet artoscópica, modificação da técnica clássica, apresenta superioridade estética e em tempo de recuperação, devendo ser gradualmente mais utilizada pelos grupos de cirurgia de ombro (GETZ CL e JOYCE CD, 2020). 
As complicações cirúrgicas nos primeiros 90 dias de acompanhamento são similares quando comparada a técnica aberta com a técnica artroscópica. A médio e a longo prazo, as complicações do tratamento cirúrgico aberto de luxação recidivante do ombro representam até $15 \%$ dos casos. A osteólise do enxerto de coracoide é uma das principais observadas em acompanhamento e, portanto, a técnica cirúrgica meticulosa e o conhecimento da anatomia local são fundamentais para melhores resultados (DOMOS P, et al., 2018; HURLEY ET, et al., 2021).

Uma revisão sistemática foi realizada no ano de 2015 agrupou diversos trabalhos comparando diferentes técnicas cirúrgicas para o tratamento da afecção estudada com a técnica de Latarjet. A revisão demonstrou a superioridade da cirurgia de Latarjet, relacionando-a a um menor índice de complicações pós-operatórias, menor necessidade de cirurgias de revisão, retorno precoce às atividades de vida diárias e esportivas. A complicação mais relacionada à cirurgia de Latarjet é a redução de amplitude do movimento de rotação externa. Em comparação com as demais técnicas foi observado que a incidência de redução do movimento de rotação do membro superior tratado com a técnica em questão foi menor (NA VVG, et al., 2015; ROSA JRP, et al., 2017).

No estudo dessa população, foram observados alguns casos que apresentarem limitação da rotação externa, apesar de esta redução da amplitude de movimento não gerar comprometimento das atividades de vida diária, em consonância com o trabalho referenciado anteriormente Em seu estudo, Rosa JRP, et al. (2017), observou a presença de alguns resultados negativos, dos quais, é interessante destacar a perda de força isométrica do músculo subescapular decorrente da tenotomia, uma etapa necessária relacionada a técnica cirúrgica utilizada.

A avaliação dos pacientes por meio do uso do questionário Carter-Rowe forneceu uma avaliação prática, menos subjetiva e de melhor aplicabilidade no estudo, de forma a poder expressar em números a qualidade de vida da pessoa perante os sintomas e sinais da luxacão recidivante do ombro. Marcondes FB, et al. (2012), em sua avaliação conseguiu realizar adaptação do questionário mundialmente utilizado para a língua portuguesa, de tal forma que fica estabelecido como uma ferramenta para o estudo realizado.

A principal limitação em relação ao trabalho foi a perda de grande parte da amostra devido à dificuldade de contato com os participantes e à falta de uma convenção de um melhor registro do escore de Carter-Rowe na primeira avaliação. Este artigo suscita novas discussões acerca da definição de qual a população mais afetada em nosso meio, do ponto de vista epidemiológico e do quão benéfico pode ser o tratamento com a técnica citada e avaliada. Estudos comparativos de outras populações se fazem necessárias para a complementação do estudo.

A mensuração da limitação de rotação externa, a comparação com outras técnicas citadas na literatura, o seguimento por um maior período, a medida de porcentagem de perda óssea, a análise sobre qual lesão óssea está presente, a presença de lesão óssea de Bankart ou de lesão de Hill-Sachs e avaliar associações com as lesões labrais são caminhos possíveis para novas pesquisas sobre este tema.

\section{CONCLUSÃO}

Diante do trabalho, foi observado que a população mais acometida compreendia pacientes do sexo masculino, adultos jovens, com idade menor que 40 anos e de trabalhadores braçais, com atividades que exigiam elevação de carga. A população da nossa amostra não era composta, em sua maioria, de pessoas que praticavam atividade esportiva. Em relação à funcionalidade, podemos concluir, a partir da avaliação dos escores, que todos os pacientes obtiveram melhora após o tratamento com a técnica de Latarjet, apesar de perda residual de rotação externa, sem impedimento a realização de atividades laborais e de vida diária, ratificando-se como opção para o tratamento da enfermidade na população estudada.

\section{AGRADECIMENTOS}

Ao Hospital Universitário, pela oportunidade de realizar esta pesquisa. Aos pacientes, que contribuíram voluntariamente para a produção científico-acadêmica. 


\section{REFERÊNCIAS}

1. AN VVG, et al. A systematic review and meta-analysis of clinical and patient-reported outcomes following two procedures for recurrent traumatic anterior instability of the shoulder: Latarjet procedure vs. Bankart repair. Journal of Shoulder and Elbow Surgery. Sydney, 2016; 25(5): 853-863.

2. BAKHSH W, NICANDRI G. Anatomy and Physical Examination of the Shoulder. Sports Med Arthrosc Rev. 2018; 26(3): e10-e22.

3. CARRAZZONE OL, et al. Prevalência das lesões associadas na luxação recidivante traumática do ombro. Revista Brasileira de Ortopedia. São Paulo, 2010; 3(46): 281-287

4. DOMOS P, et al. Contraindications and complications of the Latarjet procedure. Shoulder Elbow. 2018;10(1):15-24.

5. ESHOJ H, et al. Intertester reliability of clinical shoulder instability and laxity tests in subjects with and without selfreported shoulder problems. BMJ Open. 2018; 8(3): e018472.

6. GETZ CL, JOYCE CD. Arthroscopic Latarjet for Shoulder Instability. Orthop Clin North Am. 2020; 51(3): $373-381$.

7. GILES JW, et al. The Bristow and Latarjet Procedures: Why These Techniques Should Not Be Considered Synonymous. The Journal Of Bone And Joint Surgery. Ontario. 2014; 1340-1348.

8. HURLEY ET, et al. No difference in 90-day complication rate following open versus arthroscopic Latarjet procedure. Knee Surg Sports Traumatol Arthrosc. 2021; 29(7): 2333-2337.

9. ITOI E. 'On-track' and 'off-track' shoulder lesions. EFORT Open Rev. 2017; 2(8): 343-351.

10. LAU BC, et al. Surgical treatment for recurrent shoulder instability: factors influencing surgeon decision making. $J$ Shoulder Elbow Surg. 2021; 30(3): e85-e102.

11. LIZZIO VA, et al. Clinical Evaluation and Physical Exam Findings in Patients with Anterior Shoulder Instability. Current Reviews In Musculoskeletal Medicine, Detroit, $2017 ; 4$ (10): 434-441.

12. MARCONDES FB, et al. Tradução e adaptação cultural do Rowe Score para a língua portuguesa. Acta Ortopédica Brasileira, Campinas, 2011; 6(20): 346-350.

13. MIZUNO N, et al. Long-term results of the Latarjet procedure for anterior instability of the shoulder. Journal Of Shoulder and Elbow Surgery. Lyon, 2014. 1691-1699.

14. MORODER P, et al. Neer Award 2019: Latarjet procedure vs. iliac crest bone graft transfer for treatment of anterior shoulder instability with glenoid bone loss: a prospective randomized trial. J Shoulder Elbow Surg. 2019; 28(7): 12981307.

15. MOURA DL, et al. Modified Bristow-Latarjet procedure for treatment of recurrent traumatic anterior gleno;humeral dislocation. Revista Brasileira de Ortopedia, Coimbra, 2018; 53(2): 176-183

16. OLDS M, et al. Predicting Recurrent Instability of the Shoulder (PRIS): A Valid Tool to Predict Which Patients Will Not Have Repeat Shoulder Instability After First-Time Traumatic Anterior Dislocation. J Orthop Sports Phys Ther. 2020; 50(8): 431-437.

17. ROSA JRP, et al. Traumatic anterior instability of the shoulder. Revista Brasileira de Ortopedia, São Paulo, 2017; 5(52): 513-520.

18. SANTOS PD, et al. Padronização do estudo radiográfico da cintura escapular. Rev Bras Ortop. 1998; 33(11): 883888.

19. SILVA LA, et al. Avaliação dos resultados e das complicações em pacientes com instabilidade anterior de ombro tratados pela técnica de Latarjet. Revista Brasileira de Ortopedia, São Paulo, 2015; 50(6): 652-659.

20. WOODMASS JM, et al. Latarjet Procedure for the Treatment of Anterior Glenohumeral Instability. JBJS Essent Surg Tech. 2019; 9(3): e31.

21. WYLIE JD, et al. Functional outcomes assessment in shoulder surgery. World Journal Of Orthopedics. Salt Lake City, 2014. 623-633. 